\title{
Analysis of Adaptive MIMO Transmit Beamforming Under Channel Prediction Errors Based on Incomplete Lipschitz-Hankel Integrals
}

\author{
José F. Paris, Eduardo Martos-Naya, Unai Fernández-Plazaola, and Jesús López-Fernández
}

\begin{abstract}
This paper provides new results for a family of incomplete Lipschitz-Hankel integrals (ILHIs) which also lead to the evaluation of certain integrals involving the generalized Marcum $Q$ function. These mathematical results are then applied to analyze the bit error rate (BER) of adaptive modulation over multiple-input-multiple-output (MIMO) fading channels under imperfect channel state information (CSI). A novel exact closedform expression for the average BER of adaptive modulation under MIMO transmit beamforming with maximal ratio combining, assuming prediction errors at the receiver for the adaptation CSI required by the transmitter, is obtained. The benefit of this result with respect to previous analysis is threefold. First, the expression is an exact closed form. Second, it is applicable to any antenna configuration, and third, it allows a design improvement of the cutoff SNR thresholds, which leads to better system performance in terms of average spectral efficiency at no extra cost.
\end{abstract}

Index Terms-Adaptive modulation, channel prediction errors, exact bit error rate (BER), multiple-input-multiple-output (MIMO).

\section{INTRODUCTION}

$\mathbf{I}$ NCOMPLETE Lipschitz-Hankel integrals (ILHIs) are a class of incomplete cylindrical functions [1] that traditionally appear in the analytical solutions of numerous problems in electromagnetics [2]. Within the communication theory context, Pawula reported on the connection between the Rice $I_{e}$-function [3], which is a special type of ILHI, and the Marcum Q function [4]-[6], pointing out some advantages to be gained for performance analysis with a representation whose genesis lies in the ILHIs. However, in general, the applications of ILHIs seem to have escaped the notice of most communications theorists.

In our investigations on adaptive modulation for multipleinput-multiple-output (MIMO) fading channels with imperfect channel state information (CSI), we have encountered certain ILHIs for which, to the best of the authors' knowledge, there are no explicit expressions for their solutions in the literature, e.g., [7]-[10]. Specifically, we are interested on exact expressions for the bit error rate (BER) in terms of IHLIs of modified Bessel

Manuscript received July 3, 2008; revised October 3, 2008 and December 4, 2008. First published January 6, 2009; current version published May 29, 2009. This work was supported in part by the Spanish Government under Project TEC2007-67289/TCM and in part by AT4 wireless. The review of this paper was coordinated by Prof. C. Yuen.

The authors are with the Communication Engineering Department, Málaga University, 29071 Málaga, Spain.

Color versions of one or more of the figures in this paper are available online at http://ieeexplore.iee.org.

Digital Object Identifier 10.1109/TVT.2008.2011990 functions and a certain type of incomplete integrals involving the generalized Marcum Q function. These second types of integrals are formally equivalent to those solved in [11] and [12], whose integrands involve powers, exponentials, and the Marcum Q function with two linear arguments. However, since integration between finite limits is needed for adaptive modulation, we have to find primitives for the complete integrals solved in [11] and [12].

First, this paper shows that the incomplete integrals of the type addressed in [11] and [12] are represented by a finite number of elementary functions, generalized Marcum Q functions, and a certain family of ILHIs. Second, we prove that such ILHIs are reduced to an expression involving a finite number of modified Bessel and Marcum Q functions.

Next, we apply these results to analyze the BER of MIMO systems under imperfect CSI that employ adaptive modulation, transmit beamforming, and maximal ratio combining (MRC). This problem has been recently addressed by several authors, assuming a system model where imperfect CSI is incorporated through different assumptions [14]-[16]. In particular, we are interested in the system model first proposed in [14], where imperfect CSI originates from imperfect channel prediction performed at the receiver to be used by the transmitter for both adaptive modulation and eigenbeamforming. Interestingly, the authors obtain in [14] an approximate closed-form expression for the average BER applicable only to multiple-input-twooutput or two-input-multiple-output systems. In this paper, the mathematical tools previously developed allow the derivation of an exact closed-form expression for the average BER analysis considered in [14] applicable to system configurations with any number of antennas on each extreme. This expression can also be used for a more accurate determination of the SNR thresholds needed for adaptation.

The remainder of this paper is organized as follows. The mathematical analysis is detailed in Section II, the application to adaptive MIMO is developed in Section III, and the conclusions are given in Section IV.

\section{Mathematical Analysis}

\section{A. Definitions}

The general family of ILHIs of first-kind modified Bessel functions are defined as [13]

$$
I_{e_{\mu, \nu}}(z ; \alpha) \triangleq \int_{0}^{z} t^{\mu} e^{-\alpha, t} I_{\nu}(t) d t
$$


where $\alpha, z, \nu, \mu \in \mathbb{C}$, and to assure convergence, $\Re\{1+\mu+$ $\nu\}>0$. In particular, we are interested in the following specialization of (1).

Definition 1 (ILHI of Modified Bessel Functions):

$$
I_{e_{m, n}}(x ; \alpha) \triangleq I_{e_{\mu=m, \nu=n}}(z=x ; \alpha)
$$

where $\alpha \in \mathbb{R}, m, n \in \mathbb{N} ; \alpha>1$, and $x \in[0, \infty)$.

For simplicity, the function $I_{e_{m, n}}(x ; \alpha)$ will be referred to as the ILHI of the $m$ th degree, the $n$th order, and parameter $\alpha$. Note that Definition 1 includes the complete Lipschitz-Hankel integrals when $x \rightarrow \infty$, whose convergence is assured if $\alpha>1$ (e.g., see [10, eq. (6.624-5)]).

We are also interested in the following family of incomplete integrals involving Marcum $\mathrm{Q}$ functions (IIMQ).

Definition 2 (Incomplete Integrals of Marcum Q Functions):

$$
Q_{e_{m, n}}(x ; \beta, a, b) \triangleq \int_{0}^{x} t^{m} e^{-\beta t} Q_{n}(a \sqrt{t}, b \sqrt{t}) d t
$$

where $\beta, a, b \in \mathbb{R}, m, n \in \mathbb{N} ; \beta, a, b>0, n \geq 1$, and $x \in[0, \infty)$.

Again, for simplicity, the function $Q_{e_{m, n}}(x ; \beta, a, b)$ will be referred as the IIMQ function of the $m$ th degree, the $n$th order, and parameters $\beta, a$, and $b$. Definition 2 also includes the complete integrals solved in [11] and [12] when $x \rightarrow \infty$.

\section{B. Connection Between the IIMQ Functions and ILHIs}

We start proving that the IIMQ function can be represented by an expression involving a finite number of generalized Marcum Q functions and ILHIs.

Lemma 1: The IIMQ function can be represented as

$$
\begin{aligned}
& Q_{e_{m, n}}(x ; \beta, a, b) \\
& =\frac{m !}{\beta^{m+1}}\left\{1-e^{-\beta x} Q_{n}(a \sqrt{x}, b \sqrt{x}) \sum_{r=0}^{m} \frac{\beta^{r}}{r !} x^{r}+\frac{1}{2} \sum_{r=0}^{m} \frac{\beta^{r}}{r !}\right. \\
& \left.\times \frac{b^{n-r-1}}{a^{n+r}}\left(a I_{e_{r, n}}(a b x ; \alpha)-b I_{e_{r, n-1}}(a b x ; \alpha)\right)\right\}
\end{aligned}
$$

where $\alpha=\left(\left(a^{2}+b^{2}+2 \beta\right) / 2 a b\right)>1$.

Proof: See Appendix I.

Lemma 1 allows us to simplify our analysis, since we only need to focus on the ILHIs.

\section{Connection Between the ILHI and the Marcum Q Functions}

In this section, we will show that the ILHI can be represented by an expression involving a finite number of Bessel and Marcum Q functions. This statement is shown in Proposition 1 at the end of this section; however, to achieve this goal, we need some previous results.
Lemma 2 (Modified Sonine Identity): Let $I_{\nu}(t)$ be the $\nu$ thorder first-kind modified Bessel function and $f(t)$ be any twicedifferentiable function. Then

$$
\begin{aligned}
\int t^{\nu+1}\left\{-\frac{d^{2} f(t)}{d t^{2}}\right. & \left.-\frac{2 \nu+1}{t} \frac{d f(t)}{d t}+f(t)\right\} I_{\nu}(t) d t \\
= & t^{\nu+1}\left\{-\frac{d f(t)}{d t} I_{\nu}(t)+f(t) I_{\nu+1}(t)\right\}
\end{aligned}
$$

Proof: The steps of the proof are analogous to those followed to obtain the Sonine identity for $J_{\nu}(t)$; see [7, pp. 132] for the details.

Lemma 3: The ILHIs for low degrees and orders are given by

(i) $I_{e_{0,0}}(x ; \alpha)$

$$
\begin{aligned}
= & -2 \bar{\alpha} Q_{1}\left(\frac{\sqrt{x}}{\sqrt{\alpha}+\sqrt{\alpha^{2}-1}}, \sqrt{x} \sqrt{\alpha+\sqrt{\alpha^{2}-1}}\right) \\
& +\bar{\alpha}+\bar{\alpha} e^{-\alpha x} I_{0}(x)
\end{aligned}
$$

(ii) $I_{e_{1,0}}(x ; \alpha)$

$$
\begin{aligned}
= & -2 \alpha \bar{\alpha}^{3} Q_{1}\left(\frac{\sqrt{x}}{\sqrt{\alpha+\sqrt{\alpha^{2}-1}}}, \sqrt{x} \sqrt{\alpha+\sqrt{\alpha^{2}-1}}\right) \\
& +\alpha \bar{\alpha}^{3}+\alpha \bar{\alpha}^{3} e^{-\alpha x} I_{0}(x)-\alpha \bar{\alpha}^{2} e^{-\alpha x} x I_{0}(x) \\
& -\bar{\alpha}^{2} e^{-\alpha x} x I_{1}(x)
\end{aligned}
$$

(iii) $I_{e_{0,1}}(x ; \alpha)$

$$
\begin{aligned}
= & -2 \alpha \bar{\alpha} Q_{1}\left(\frac{\sqrt{x}}{\sqrt{\alpha+\sqrt{\alpha^{2}-1}}}, \sqrt{x} \sqrt{\alpha+\sqrt{\alpha^{2}-1}}\right) \\
& +\alpha \bar{\alpha}-1+(1+\alpha \bar{\alpha}) e^{-\alpha x} I_{0}(x)
\end{aligned}
$$

(iv) $I_{e_{1,1}}(x ; \alpha)$

$$
\begin{aligned}
= & -2 \bar{\alpha}^{3} Q_{1}\left(\frac{\sqrt{x}}{\sqrt{\alpha+\sqrt{\alpha^{2}-1}}}, \sqrt{x} \sqrt{\alpha+\sqrt{\alpha^{2}-1}}\right) \\
& +\bar{\alpha}^{3}+\bar{\alpha}^{3} e^{-\alpha x} I_{0}(x)-\bar{\alpha}^{2} e^{-\alpha x} x I_{0}(x) \\
& -\alpha \bar{\alpha}^{2} e^{-\alpha x} x I_{1}(x)
\end{aligned}
$$

where $\bar{\alpha} \triangleq 1 / \sqrt{\alpha^{2-1}}$.

Proof: See Appendix II.

Now, we are in a position to show that the $m$ th-degree $n$ thorder ILHI fits the pattern exhibited by $I_{e_{0,0}}, I_{e_{1,0}}, I_{e_{0,1}}$, and $I_{e_{1,1}}$ in Lemma 3.

Proposition 1: The $m$ th-degree $n$ th-order ILHI is represented by an expression involving a first-order Marcum Q function and a finite number of Bessel functions, specifically

$$
\begin{aligned}
& I_{e_{m, n}}(x ; \alpha)=\mathcal{A}_{m, n}^{0}(\alpha)+e^{-\alpha x} \sum_{i=0}^{m} \sum_{j=0}^{n+1} \mathcal{B}_{m, n}^{i, j}(\alpha) x^{i} I_{j}(x) \\
& \quad+\mathcal{A}_{m, n}^{1}(\alpha) Q_{1}\left(\frac{\sqrt{x}}{\sqrt{\alpha+\sqrt{\alpha^{2}-1}}}, \sqrt{x} \sqrt{\alpha+\sqrt{\alpha^{2}-1}}\right)
\end{aligned}
$$

where the set of coefficients $\mathcal{A}_{m, n}^{l}(\alpha), \mathcal{B}_{m, n}^{i, j}(\alpha)$ can be recursively obtained. 


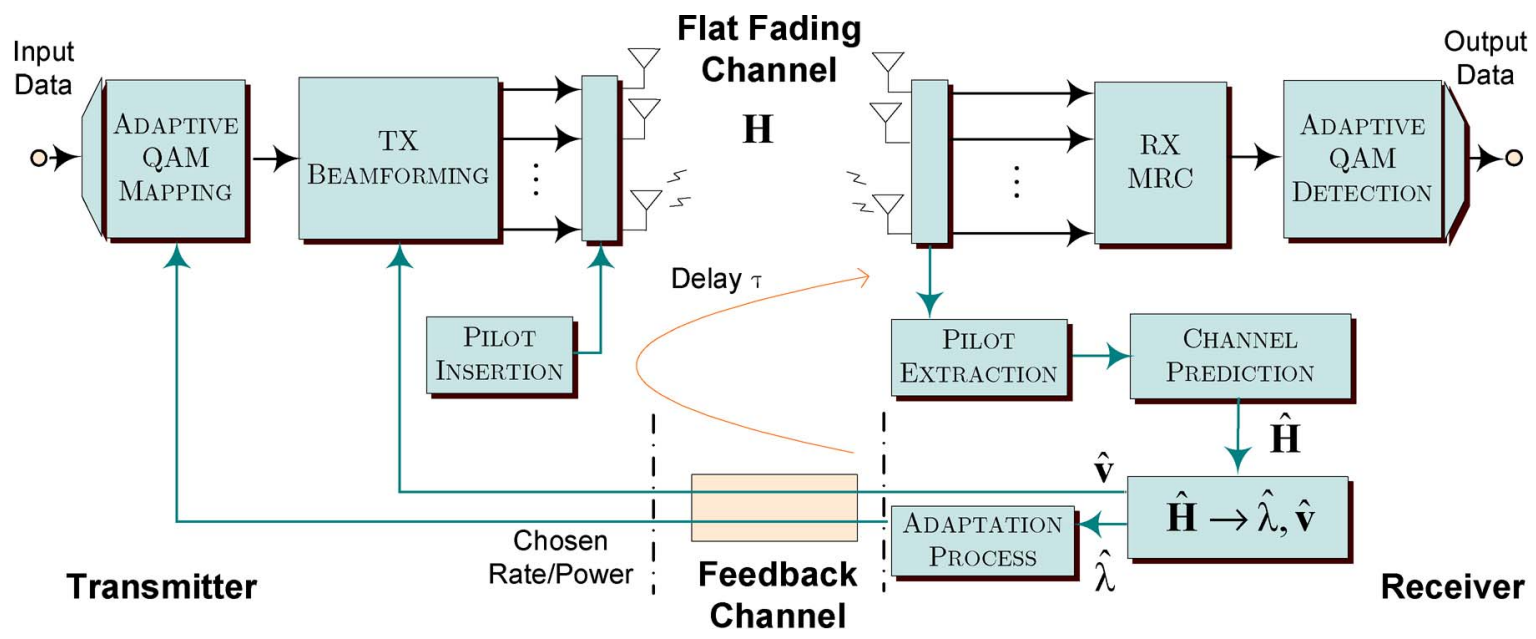

Fig. 1. System model for adaptive MIMO transmit beamforming with MRC and prediction errors.

Proof: See Appendix III.

From Lemma 1 and Proposition 1, we readily obtain a useful representation of the IIMQ function.

Corollary 1: The $m$ th-degree $n$ th-order IIMQ is represented by the following expression involving a finite number of Marcum Q and Bessel functions:

$$
\begin{aligned}
& Q_{e_{m, n}}(x ; \beta, a, b) \\
& =-\frac{m !}{\beta^{m+1}} e^{-\beta x} Q_{n}(a \sqrt{x}, b \sqrt{x}) \sum_{r=0}^{m} \frac{\beta^{r}}{r !} x^{r} \\
& +\frac{m !}{\beta^{m+1}}+\frac{1}{2} \frac{m !}{\beta^{m+1}} \sum_{r=0}^{m} \frac{\beta^{r}}{r !} \frac{b^{n-r-1}}{a^{n+r}} \\
& \times\left\{a \mathcal{A}_{r, n}^{0}(\alpha)+\left(a \mathcal{A}_{r, n}^{1}(\alpha)-b \mathcal{A}_{r, n-1}^{1}(\alpha)\right) Q_{1}\right. \\
& \quad \times\left(\frac{\sqrt{a b x}}{\xi}, \sqrt{a b x} \xi\right)-b \mathcal{A}_{r, n-1}^{0}(\alpha) \\
& \quad+e^{-\alpha a b x} \sum_{i=0}^{r} a \mathcal{B}_{r, n}^{i, n+1}(\alpha)(a b x)^{i} I_{n+1}(a b x) \\
& \left.\quad+\sum_{j=0}^{n}\left(a \mathcal{B}_{r, n}^{i, j}(\alpha)-b \mathcal{B}_{r, n-1}^{i, j}(\alpha)\right)(a b x)^{i} I_{j}(a b x)\right\}
\end{aligned}
$$

where $\alpha=\left(a^{2}+b^{2}+2 \beta\right) / 2 a b$, and $\xi=\sqrt{\alpha+\sqrt{\alpha^{2}-1}}$.

The finite recursive algorithm to obtain the coefficients of Proposition 1 and Corollary 1 is found in Appendix III.

\section{Applichtion to Adaptive MiMO Analysis}

The mathematical tools developed in the previous section are useful to carry out performance analysis of several interesting adaptive MIMO systems. In this section, we present, as an application case, an exact closed-form BER analysis of MIMO transmit beamforming with MRC under imperfect CSI at the transmitter for systems performing adaptive modulation over Rayleigh fading channels and with any antenna configuration.

\section{A. System Model}

The system model for adaptive MIMO transmit beamforming with MRC is depicted in Fig. 1. A detailed description of the adopted system model can be found in [14].

In short, we consider $N_{T}$ transmit antennas and $N_{R}$ receive antennas, and the channel gain is modeled by an $N_{R} \times N_{T}$ complex matrix $\mathbf{H}$ so that each entry $H_{i, j}$ is the channel coefficient between the $j$ th transmit and the $i$ th receive antenna. These channel coefficients exhibit frequency-flat slowly timevarying fading. The entries $H_{i, j}$ are assumed to be independent identically distributed (i.i.d.) complex circularly symmetric normal random variables (RVs), with zero mean and unity variance, i.e., $H_{i, j} \sim \mathcal{C N}(0,1)$, where the symbol $\sim$ means statistically distributed as. Noise is modeled by an additive $N_{R}$-dimensional vector $\mathbf{n}$, whose entries $n_{k}$ are i.i.d. complex circularly symmetric normal $\mathrm{RVs} \sim \mathcal{C N}\left(0, \sigma_{n}^{2}\right)$. The received signal can be expressed as

$$
\mathbf{y}=\mathbf{H x}+\mathbf{n}
$$

where $\mathbf{y}$ is the received $N_{R}$-dimensional complex vector, and $\mathbf{x}$ is the transmitted $N_{T}$-dimensional complex vector.

At the receiver, the channel matrix $\mathbf{H}$ is predicted $\tau$ s ahead to perform adaptive modulation and eigenbeamforming at the transmitter. The channel prediction $\hat{\mathbf{H}}$ can be expressed as follows:

$$
\hat{\mathbf{H}}=\mathbf{H}-\hat{\boldsymbol{\Xi}}, \text { with } \begin{cases}\hat{\mathrm{H}}_{i, j}, & \text { i.i.d. } \mathrm{RVs} \sim \mathcal{C} N(0,1-\chi) \\ \hat{\Xi}_{i, j}, & \text { i.i.d. } \mathrm{RVs} \sim \mathcal{C} N(0, \chi)\end{cases}
$$

where $\hat{\boldsymbol{\Xi}}$ is the prediction error matrix in which entries and their corresponding ones in $\hat{\mathbf{H}}$ are mutually orthogonal. Finally, $\chi$ represents the prediction minimum mean square error.

To maximize the received SNR, the beam-steering vector $\hat{\mathbf{v}}$ employed at the transmitter is chosen as the $N_{T}$-dimensional eigenvector corresponding to the largest eigenvalue $\hat{\lambda}$ of matrix $\hat{\mathbf{H}}^{\mathcal{H}} \hat{\mathbf{H}}$, which is given by $\hat{\lambda}=\hat{\mathbf{v}}^{\mathcal{H}} \hat{\mathbf{H}}^{\mathcal{H}} \hat{\mathbf{H}} \hat{\mathbf{v}}$. In each frame, the receiver feeds the vector $\hat{\mathbf{v}}$ back to the transmitter to perform beamforming so that the transmitted vector becomes $\mathbf{x}=\hat{\mathbf{v}} z$, where $z$ is the complex transmitted symbol. The effective channel vector is $N_{R}$-dimensional and is defined as $\mathbf{h} \triangleq \mathbf{H} \hat{\mathbf{v}}$, and 
the predicted effective channel vector is the vector $\hat{\mathbf{h}} \triangleq \hat{\mathbf{H}} \hat{\mathbf{v}}$, whose square Euclidean norm is $\|\hat{\mathbf{h}}\|^{2}=\hat{\lambda}$. The effective channel vector can also be expressed as $\mathbf{h} \triangleq \mathbf{H} \hat{\mathbf{v}}=(\hat{\mathbf{H}}+\hat{\boldsymbol{\Xi}}) \hat{\mathbf{v}}=$ $\hat{\mathbf{h}}+\boldsymbol{\Psi}$, where $\boldsymbol{\Psi}$ is a complex normal $N_{R}$-dimensional vector whose entries $\Psi_{k}$, assuming that $\hat{\mathbf{v}}$ is a unitary vector, are i.i.d. $\mathrm{RVs} \sim \mathcal{C N}(0, \chi)[18$, pp. 26].

The receiver must estimate the current effective channel vector $\mathbf{h}$ to perform MRC. Assuming perfect estimation of $\mathbf{h}$ at the receiver (as in [14]), the symbol $r$ that results from applying MRC to received vector $\mathbf{y}$ is given by

$$
r \triangleq \frac{\mathbf{h}^{\mathcal{H}} \mathbf{y}}{\|\mathbf{h}\|^{2}}=z+\frac{\mathbf{h}^{\mathcal{H}} \mathbf{n}}{\|\mathbf{h}\|^{2}}=z+\frac{(\hat{\mathbf{h}}+\mathbf{\Psi})^{\mathcal{H}} \mathbf{n}}{\|\hat{\mathbf{h}}+\mathbf{\Psi}\|^{2}}=z+\zeta
$$

where $\zeta$ is the resultant noise after MRC.

Finally, the largest eigenvalue $\hat{\lambda}$ is employed to perform adaptive modulation. Consistently with the single-input-singleoutput case, we define the predicted instantaneous SNR as $\widehat{\gamma} \triangleq \hat{\lambda} \bar{\gamma}$, where $\bar{\gamma} \triangleq\left(\bar{S}_{T} / \sigma_{n}^{2}\right)$ is the average SNR, and $\bar{S}_{T}$ is the average transmit power. We assume a generalized adaptation policy that employs a set of $N_{F}$ prefixed rates $\left\{R_{i}\right\}_{i=0}^{N_{F}-1}$ and powers per constellation $\left\{S_{i}\right\}_{i=0}^{N_{F}-1}$ (including $R_{0}=S_{0}=0$ as NOTX), i.e., this scheme considers $N_{F}$ fading regions. Within the $i$ th fading region that spans from the lower SNR switching threshold $\widehat{\gamma}_{i-1}$ to the upper threshold $\widehat{\gamma}_{i}$, the rate $R_{i}$ is employed using BPSK or square $2^{R_{i}}$-quadratic-amplitudemodulation (QAM) constellations with power $S_{i}$. For notation simplicity, we assume that $\widehat{\gamma}_{-1} \triangleq 0$ and $\widehat{\gamma}_{N_{F}-1} \triangleq \infty$. Note that constant-power policies [19] are also included in our generalized adaptation policy, assuming that above the cutoff threshold $\widehat{\gamma}_{0}$, transmission is carried out using constant power, i.e., $S_{i}=S_{1}, \forall i=1, \ldots, N_{F}-1$.

\section{B. BER Analysis}

The exact expression for the BER is

$$
\mathrm{BER}=\frac{1}{\bar{R}} \sum_{i=1}^{N_{F}-1} R_{i} \int_{\hat{\gamma}_{i-1}}^{\hat{\gamma}_{i}} \operatorname{CBER}\left(\widehat{\gamma} ; R_{i}, S_{i}\right) p_{\hat{\gamma}}(\hat{\gamma}) d \hat{\gamma}
$$

with

$$
\bar{R} \triangleq \sum_{i=1}^{N_{F}-1} R_{i} \int_{\hat{\gamma}_{i-1}}^{\hat{\gamma}_{i}} p_{\hat{\gamma}}(\hat{\gamma}) d \hat{\gamma}
$$

where $p_{\hat{\gamma}}(\hat{\gamma})$ is the probability density function (pdf) of $\hat{\gamma}$, $\operatorname{CBER}\left(\widehat{\gamma} ; R_{i}, S_{i}\right)$ is the BER conditioned to $\hat{\gamma}$, and $\bar{R}$ is the average data rate (in bits per complex symbol).

The pdf of the largest eigenvalue of complex Wishart matrices can be expressed as a sum of elementary Gamma pdfs [17]. Thus, the pdf of the predicted instantaneous SNR is given by

$$
p_{\hat{\gamma}}(\hat{\gamma})=B_{m, n} \sum_{k=1}^{m} \sum_{l=n-m}^{(n+m-2 k) k} \frac{A_{k, l} \hat{\gamma}^{l}}{((1-\chi) \bar{\gamma})^{l+1}} \exp \left(-\frac{k \hat{\gamma}}{(1-\chi) \bar{\gamma}}\right)
$$

where $m \doteq \min \left\{N_{T}, N_{R}\right\}, \quad n \doteq \max \left\{N_{T}, N_{R}\right\}, \quad B_{m, n}=$ $\left(\prod_{i=1}^{m}(m-i) ! \prod_{i=1}^{m}(n-i) !\right)^{-1}$, and the coefficients $A_{k, l}$ are determined by the algorithm proposed in [17].

From the pdf of the predicted instantaneous SNR, the average data rate is exactly computed, yielding [15]

$$
\begin{aligned}
\bar{R} & =\sum_{i=1}^{N_{F}-1} R_{i} \sum_{k=1}^{m} \sum_{l=n-m}^{(n+m-2 k) k} \sum_{w=0}^{l} \frac{l ! A_{k, l} B_{m, n}}{w ! k^{l+1}} \\
& \times\left[\left(\frac{k \hat{\gamma}_{i-1}}{(1-\chi) \bar{\gamma}}\right)^{w} e^{-\frac{k \hat{\gamma}_{i-1}}{(1-\chi) \bar{\gamma}}}-\left(\frac{k \hat{\gamma}_{i}}{(1-\chi) \bar{\gamma}}\right)^{w} e^{-\frac{k \hat{\gamma}_{i}}{(1-\chi) \bar{\gamma}}}\right] .
\end{aligned}
$$

Our goal is to obtain an exact closed-form expression for (11) in terms of standard special functions. For this task, a closedform expression for the conditional BER (CBER) is required, and incomplete integration must be performed.

Next, Section III-B1 summarizes the results obtained in [14] concerning (11), where the closed-form expression for the CBER is obtained by exponential-type approximations, and an expression involving incomplete Gamma functions is achieved by incomplete integration. Section III-B2 shows that starting from exact closed-form expressions for the CBER in terms of Bessel and Marcum Q functions, an exact closedform expression for (11) involving ILHIs and IIMQs can be obtained.

1) Approximate BER Expressions: Obtaining an exact closed-form expression for the incomplete integrals involving the CBER that appear in (11) is cumbersome. Consequently, to analyze the BER, the following approximation for the CBER was obtained in [14, eq. (38)] (up to notation):

$$
\operatorname{CBER}(\widehat{\gamma} ; R, S) \approx \frac{1}{5 \Phi^{N_{R}}} \exp \left(\frac{-3}{2} \frac{1}{\Phi} \frac{S}{\bar{S}_{T}} \frac{\hat{\gamma}}{2^{R}-1}\right)
$$

where

$$
\Phi \triangleq 1+\frac{8}{5} \frac{S}{\bar{S}_{T}} \frac{\chi \bar{\gamma}}{2^{R}-1} .
$$

Integrating (14) with the pdf (12) is carried out by incomplete Gamma functions; thus, an approximate expression in closed form for the system BER is obtained [14, eq. (46)].

2) Exact BER Expressions: With the mathematical tools summarized in Proposition 1 and Corollary 1 in hand, we are now in a position to obtain an exact closed-form expression of the system BER.

To achieve this goal, we have to start from exact closedform expressions for the CBER of BPSK and square $M$-QAM under prediction errors. For these signaling formats, we define the error probability conditioned on a predicted channel state (CCEP) as

$$
\mathcal{I}(p ; \hat{\gamma}) \triangleq \operatorname{Pr}\{\Re\{\zeta\}>(2 p-1) d \mid \hat{\gamma}\}
$$

which represents the probability that the real projection of the noise after MRC, i.e., $\zeta$, surpasses the symbol-to-decisionboundary distance $(2 p-1) d$, where $p$ is a positive integer, and $2 d$ is the minimum distance between constellation symbols. The 
CBER under prediction errors when the usual Gray mapping is adopted can be exactly computed by

$$
\operatorname{CBER}(\hat{\gamma} ; R, S)=C \sum_{p=1}^{L-1} \omega(p) \mathcal{I}(p ; \hat{\gamma})
$$

where $C=(1 / R L)$, and $L=2^{R / 2}$ for square $2^{R}$-QAM, $C=$ $1 / 2$ and $L=2$ for BPSK, and $\omega(p)$ are certain integers' coefficients. For BPSK, $\omega(p)=2$, whereas for $M$-QAM, these coefficients can be computed explicitly using [20].

The CCEP terms in (17) can be exactly calculated in closed form using the quadratic-form-based method proposed by Proakis [21, App. B] (see [22] for details)

$$
\begin{aligned}
& I(p ; \hat{\gamma})=Q_{1}\left(a\left(\theta_{p}\right) \sqrt{\frac{\hat{\gamma}}{\chi \bar{\gamma}}}, b\left(\theta_{p}\right) \sqrt{\frac{\hat{\gamma}}{\chi \bar{\gamma}}}\right)+\sum_{q=0}^{N_{R}-1} D_{q}\left(\eta\left(\theta_{p}\right) ; N_{R}\right) \\
& \quad \times I_{q}\left(a\left(\theta_{p}\right) b\left(\theta_{p}\right) \frac{\hat{\gamma}}{\chi \bar{\gamma}}\right) \exp \left(-\frac{a^{2}\left(\theta_{p}\right)+b^{2}\left(\theta_{p}\right)}{2} \frac{\hat{\gamma}}{\chi \bar{\gamma}}\right)
\end{aligned}
$$

where $Q_{1}$ is the Marcum Q function, $I_{q}$ is the $q$ th-order modified Bessel function of the first kind, $\theta_{p} \triangleq\left(3(2 p-1)^{2} / 2\left(2^{R}-\right.\right.$ 1)) $\bar{\gamma} \chi S / \bar{S}$ for $M$-QAM, and $\theta_{p} \triangleq(2 p-1)^{2} \bar{\gamma} \chi S / \bar{S}$ for BPSK; the functions $\eta(\cdot), a(\cdot)$, and $b(\cdot)$ are defined for any nonnegative real as

$$
\begin{aligned}
& \eta(x) \triangleq 1+2 x+2 \sqrt{x(1+x)} \\
& a(x) \triangleq \sqrt{2} \frac{1}{1+\eta(x)} \\
& b(x) \triangleq \sqrt{2} \frac{\eta(x)}{1+\eta(x)}
\end{aligned}
$$

whereas the function $D_{q}\left(x ; N_{R}\right)$ is defined for any nonnegative real as follows:

$$
D_{q}\left(x ; N_{R}\right) \triangleq-1+\frac{1}{(1+\eta(x))^{2 N_{R}-1}} \sum_{k=0}^{N_{R}-1}\left(\begin{array}{c}
2 N_{R}-1 \\
k
\end{array}\right) \eta(x)^{k}
$$

for $q=0$, and

$$
\begin{array}{r}
D_{q}\left(x ; N_{R}\right) \triangleq \frac{1}{(1+\eta(x))^{2 N_{R}-1}} \sum_{k=0}^{N_{R}-1-q}\left(\begin{array}{c}
2 N_{R}-1 \\
k
\end{array}\right) \\
\times\left[\eta(x)^{k+q}-\eta(x)^{2 N_{R}-1-k-q}\right]
\end{array}
$$

for $0<q \leqslant N_{R}-1$.

At this point, the natural question that arises is the connection between the well-known simple exponential approximation (14) and the more complicated exact expression given in (17)-(20). Note that (14) is derived from [19, eq. (9.7)], which is only a tight bound under certain conditions; specifically, it is quite inaccurate for low instantaneous SNR $\hat{\gamma}$. Despite being complicated, the expression derived above is exact and can provide insight into the system performance when a concrete scenario is considered, e.g., using asymptotic analysis as in [23]. In particular, let us consider $M$-QAM under $\chi \bar{\gamma} \ll 1$,

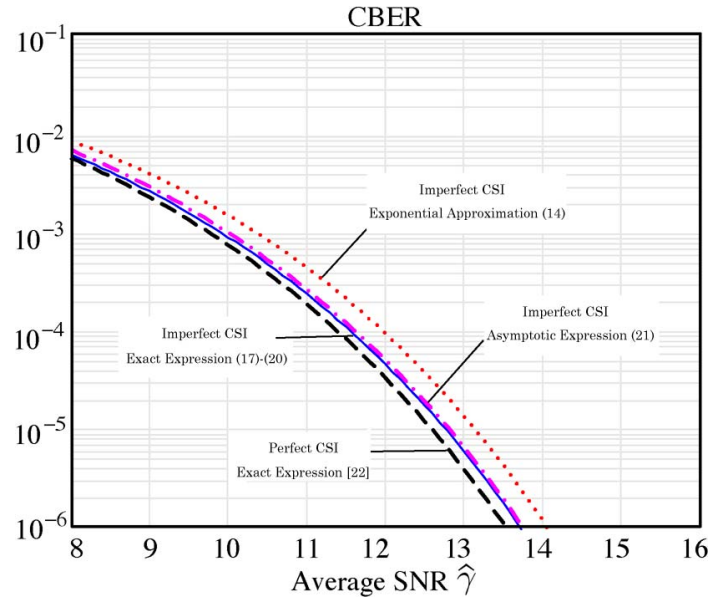

Fig. 2. CBER for $\chi \bar{\gamma}=0.1, R=2, S=S_{T}$, and $N_{R}=2$ using different expressions.

which corresponds to a good but not perfect CSI. Then, it is shown in Appendix IV that

$$
\begin{aligned}
\operatorname{CBER}(\hat{\gamma} ; R, S) \sim & \frac{1}{R 2^{R / 2}} \sum_{p=1}^{L-1} \omega(p) \frac{1}{\sqrt{\Delta S(p)}} \\
& \times Q\left(\sqrt{\Delta S(p)} \sqrt{\frac{3(2 p-1)^{2}}{\left(2^{r}-1\right)}} \frac{S}{\bar{S}} \hat{\gamma}\right)
\end{aligned}
$$

as $\quad \hat{\gamma} \rightarrow \infty$, where $\Delta S(p)=\left(1+\left(3(2 p-1)^{2} /\left(2^{R}-\right.\right.\right.$ 1)) $\chi \bar{\gamma}(S / \bar{S}))^{-1}$, and $Q$ is the Gaussian $Q$-function. When $\Delta S(p)=0,(21)$ provides the CBER for perfect CSI [22]; thus, $\Delta S(p)$ represents the limiting power penalty due to imperfect CSI. As shown in Fig. 2 for $\chi \bar{\gamma}=0.1, R=2, S=S_{T}$, and $N_{R}=2$, the asymptotic expression (21) is quite close to the exact expression, while the exponential approximation (14) exhibits an irreducible gap, even for an arbitrarily high instantaneous SNR.

The BER is derived as follows. Introducing (18) in (17) and the result in (11), we obtain

$$
\begin{aligned}
\mathrm{BER}= & \frac{1}{\bar{R}} \sum_{i=1}^{N_{F}-1} R_{i} \int_{\hat{\gamma}_{i-1}}^{\hat{\gamma}_{i}} \operatorname{CBER}\left(\hat{\gamma} ; R_{i}, S_{i}\right) p_{\hat{\gamma}}(\hat{\gamma}) d \hat{\gamma} \\
= & \frac{1}{\bar{R}} \sum_{i=1}^{N_{F}-1} \sum_{k=1}^{m} \sum_{l=n-m}^{(n+m-2 k) k} \sum_{p=1}^{L-1} \frac{R_{i} A_{k, l} B_{m, n} C_{i} \omega_{i}(p)}{((1-\chi) \bar{\gamma})^{l+1}} \\
& \times\left\{\int_{\hat{\gamma}_{i-1}}^{\hat{\gamma}_{i}} \hat{\gamma}^{l} \exp \left(-\frac{k}{(1-\chi)} \frac{\hat{\gamma}}{\bar{\gamma}}\right)\right. \\
& \times Q_{1}\left(a\left(\theta_{p, i}\right) \sqrt{\frac{\hat{\gamma}}{\chi \bar{\gamma}}}, b\left(\theta_{p, i}\right) \sqrt{\frac{\hat{\gamma}}{\chi \bar{\gamma}}}\right) d \hat{\gamma} \\
& +\sum_{q=0}^{N_{R}-1} D_{q}\left(\theta_{p, i} ; N_{R}\right) \int_{\hat{\gamma}_{i-1}}^{l} I_{q}\left(a\left(\theta_{p, i}\right) b\left(\theta_{p, i}\right) \frac{\hat{\gamma}}{\chi \bar{\gamma}}\right) \\
& \left.\times \exp \left(-\left[\frac{k \chi}{(1-\chi)}+\frac{a^{2}\left(\theta_{p, i}\right)+b^{2}\left(\theta_{p, i}\right)}{2}\right] \frac{\hat{\gamma}}{\bar{\gamma} \chi}\right) d \hat{\gamma}\right\}
\end{aligned}
$$


where $L_{i}, C_{i}, \omega_{i}(p)$, and $\theta_{p, i}$ represent the values of $L, C$, $\omega(p)$, and $\theta_{p}$, respectively, within the $i$ th fading region where the rate $R_{i}$ and power $S_{i}$ are employed. In (22), we find two types of integrals that resemble those analyzed in the first part of the paper. It is straightforward to check that both types of integrals fit our definitions. Concretely, with the simple change of variable $t=a\left(\theta_{p, i}\right) b\left(\theta_{p, i}\right)(\hat{\gamma} / \chi \bar{\gamma})$ in the second integral and after some algebra, we can reach

$$
\begin{aligned}
\mathrm{BER}= & \frac{1}{\bar{R}} \sum_{i=1}^{N_{F}-1} R_{i} \int_{\hat{\gamma}_{i-1}}^{\hat{\gamma}_{i}} \operatorname{CBER}\left(\hat{\gamma} ; R_{i}, S_{i}\right) p_{\hat{\gamma}}(\hat{\gamma}) d \hat{\gamma} \\
= & \frac{1}{\bar{R}} \sum_{i=1}^{N_{F}-1} \sum_{k=1}^{m} \sum_{l=n-m}^{(n+m-2 k) k} \sum_{p=1}^{L-1} \frac{R_{i} A_{k, l} B_{m, n} C_{i} \omega_{i}(p)}{((1-\chi) \bar{\gamma})^{l+1}} \\
& \times\left\{Q_{e_{l, 1}}\left(\hat{\gamma}_{i} ; \frac{k}{(1-\chi) \bar{\gamma}}, \frac{a\left(\theta_{p, i}\right)}{\sqrt{\chi \bar{\gamma}}}, \frac{b\left(\theta_{p, i}\right)}{\sqrt{\chi \bar{\gamma}}}\right)\right. \\
& -Q_{e_{l, 1}}\left(\hat{\gamma}_{i-1} ; \frac{k}{(1-\chi) \bar{\gamma}}, \frac{a\left(\theta_{p, i}\right)}{\sqrt{\chi \bar{\gamma}}}, \frac{b\left(\theta_{p, i}\right)}{\sqrt{\chi \bar{\gamma}}}\right) \\
& +\sum_{q=0}^{N_{R}-1} D_{q}\left(\theta_{p, i} ; N_{R}\right)\left(\frac{\chi \bar{\gamma}}{a\left(\theta_{p, i}\right) b\left(\theta_{p, i}\right)}\right) \\
& \left.\times\left[I_{e_{l, q}}\left(t_{p, i}^{+} ; \alpha_{p, i, k}\right)-I_{e_{l, q}}\left(t_{p, i}^{-} ; \alpha_{p, i, k}\right)\right]\right\}
\end{aligned}
$$

where

$$
\left\{\begin{array}{l}
t_{p, i}^{+} \triangleq a\left(\theta_{p, i}\right) b\left(\theta_{p, i}\right) \frac{\hat{\gamma}_{i}}{\chi \bar{\gamma}} \\
t_{p, i}^{-} \triangleq a\left(\theta_{p, i}\right) b\left(\theta_{p, i}\right) \frac{\hat{\gamma}_{i-1}}{\chi \bar{\gamma}} \\
\alpha_{p, i, k} \triangleq \frac{k \chi}{(1-\chi) a\left(\theta_{p, i}\right) b\left(\theta_{p, i}\right)}+\frac{a^{2}\left(\theta_{p, i}\right)+b^{2}\left(\theta_{p, i}\right)}{2 a\left(\theta_{p, i}\right) b\left(\theta_{p, i}\right)} .
\end{array}\right.
$$

Note that (23) is a compact expression of the system BER in terms of the ILHI and IIMQ analyzed in the first part of the paper. Using Proposition 1 and Corollary 1, the expression (23) of the BER can be directly expanded and reexpressed in terms of a finite number of Marcum Q, Modified Bessel, and algebraic functions. Expression (23) has two basic advantages with respect to the approximate BER expression obtained in [14, eq. (46)]: 1) (23) is an exact closed-form expression for the same system model, and 2) it is valid for any antenna configuration, whereas in [14, eq. (46)], only multiple-input-twooutput or two-input-multiple-output systems are addressed. Moreover, (23) allows for a more accurate calculation of the SNR switching thresholds used in the adaptation, which turns into a better spectral efficiency, as is shown below.

\section{Adaptation Policy}

The detailed design of adaptation policies is out of the scope of this paper. A nonexhaustive list of references on this topic is [19], [24]-[32], where [29]-[32] specifically address the design under imperfect CSI.

To confirm the potential of our analytical results, we consider a particular adaptation policy that employs constant power $\left\{S_{i}=\bar{S}_{T}\right\}_{i=1}^{N_{F}-1}$ and the usual set of rates $\left\{R_{i}\right\}_{i=1}^{N_{F}-1}=$
$1,2,4,6, \ldots$, which correspond to BPSK or square $2^{R_{i}}$-QAM constellations. The SNR switching thresholds are set to follow the relation $\hat{\gamma}_{i}=\left(2^{R_{i}}-1\right) \hat{\gamma}_{0}$ for $i=1, \ldots, N_{F}-2$ [24], where the value of the SNR cutoff threshold $\hat{\gamma}_{0}$ will be calculated to meet the BER constraint.

Introducing this particularization in the exact expression for the BER in (11) results in

$$
\operatorname{BER}\left(\hat{\gamma}_{0}\right)=\frac{\sum_{i=1}^{N_{F}-1} R_{i} \int_{\left(2^{R_{i-1}}-1\right) \hat{\gamma}_{0}}^{\left(2^{R_{i}}-1\right) \hat{\gamma}_{0}} \operatorname{CBER}\left(\hat{\gamma} ; R_{i}, S_{i}\right) p_{\hat{\gamma}}(\hat{\gamma}) d \hat{\gamma}}{\sum_{i=1}^{N_{F}-1} R_{i} \int_{\left(2^{\left.R_{i-1}-1\right)} \hat{\gamma}_{0}\right.}^{\left(2^{R_{i}}-1\right) \hat{\gamma}_{0}} p_{\hat{\gamma}}(\hat{\gamma}) d \hat{\gamma}} .
$$

Given a target BER, hereinafter referred to as $\mathrm{BER}_{T}$, the SNR cutoff threshold $\hat{\gamma}_{0}$ (and from it, the rest of the thresholds) is obtained by solving the equation $\operatorname{BER}\left(\hat{\gamma}_{0}\right)=\mathrm{BER}_{T}$.

\section{Results}

In this section, optimal Wiener finite-impulse response (FIR) filtering for channel prediction is assumed, using the MIMO extension of classical pilot-symbol-assisted-modulation; see [14] for details. Channel time variation is assumed to follow the well-known Jake's model. It is also assumed that the data stream is parsed into frames of duration of $P T_{S} \mathrm{~s}$, where $T_{S}$ is the symbol interval, and $P$ is the frame length. A known pilot symbol is inserted within each frame along $N_{T}$ symbol intervals; thus, the average spectral efficiency (ASE) $\bar{\nu}$ has a $\left(P-N_{T}\right) / P$ penalty factor, i.e., $\bar{\nu} \triangleq\left(P-N_{T} / P\right) \bar{R}$. The general system parameters used to obtain the results shown in the subsequent figures are 1) carrier frequency of $3 \mathrm{GHz}$; 2) symbol frequency $1 / T_{S}=100 \mathrm{kHz} ; 3$ ) pilot insertion interval $P=64$; 4) adaptation delay $\tau=2 T_{S} P$; 5) pilot symbol SNR $\bar{\gamma}_{P}=\bar{\gamma} ; 6$ ) the number of taps of the prediction FIR filter $F=8 ; 7)$ the number of fading regions $N_{F}=5$; and 8) target BER $\mathrm{BER}_{T}=10^{-3}$. From this set of system parameters, the prediction error $\chi$ is obtained as indicated in [14].

The next three sections are aimed to show the performance of the exact closed-form expression for the BER in (23). In Section III-D1, the BER of a certain MIMO system is calculated with our exact expression (23) and with the approximate formula in [14, eq. (46)]. Simulation results show the accuracy of our BER expression. Section III-D2 shows the improvement in the determination of the set of SNR thresholds that can be achieved if (23) is used. Finally, in Section III-D3, the applicability of (23) to analyze systems beyond merely the two-input-multiple-output or multipleinput-two-output antenna configuration is demonstrated.

1) Evaluation of the BER: Fig. 3 shows the BER in terms of the average SNR $\bar{\gamma}$ for an adaptive MIMO transmitbeamforming $2 \times 2$ system with MRC. The cutoff SNR threshold is fixed according to the well-known expression $\hat{\gamma}_{0}=-(2 / 3) \log \left(5 \mathrm{BER}_{T}\right)$ [24], [25]. Three scenarios employing different mobile speeds $v$ are considered. Note that the BER is significantly different for the approximation [14, eq. (46)] and for the exact expression (23). The plot for the BER approximation [14, eq. (46)] is clearly above that of the 


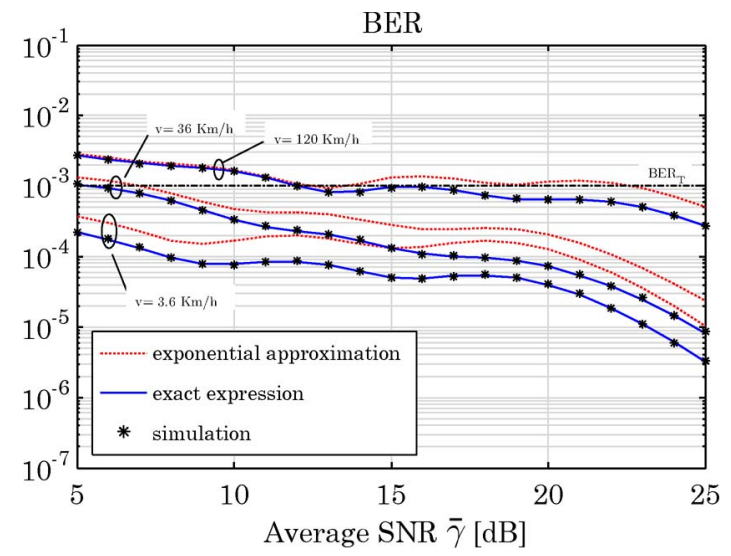

Fig. 3. Exact, approximate, and simulated BERs. Adaptive $2 \times 2$ MIMO transmit beamforming with MRC, $N_{F}=5$, and $\mathrm{BER}_{T}=10^{-3}$.

exact BER, as it is based on exponential upper bounds for the error probability in Gaussian channels [24]. Also superimposed in the figure are given simulation results, which serve as a check for our analytical results.

2) Improvement in the Design of the SNR Thresholds: Consider two sets of SNR thresholds obtained by solving the equation for the BER constraint

$$
\operatorname{BER}\left(\hat{\gamma}_{0}\right)=\operatorname{BER}_{T}
$$

The first set, which is denoted by $\left\{\hat{\gamma}_{i}^{\nabla}\right\}$, corresponds to the solution of (26) when the exponential-approximation-based CBER expression (14) is substituted in the numerator of (25). The second set, which is denoted as $\left\{\hat{\gamma}_{i}^{\Delta}\right\}$, corresponds to the solution of (26) when the exact CBER expressions (17) and (18) are used in the numerator of (25).

Fig. 4 shows the ASE and the BER in terms of the average SNR $\bar{\gamma}$ for an adaptive MIMO transmit-beamforming $2 \times 2$ system with MRC, mobile speed $v=36 \mathrm{Km} / \mathrm{h}$, and two sets of thresholds. As can be seen, a significant performance improvement is achieved when the set of SNR thresholds $\left\{\hat{\gamma}_{i}^{\Delta}\right\}$ (based on the exact CBER expression) is employed instead of the set $\left\{\hat{\gamma}_{i}^{\nabla}\right\}$ (based on the approximate CBER expression). Note in Fig. 4(a) that using $\left\{\hat{\gamma}_{i}^{\Delta}\right\}$, the BER meets $\operatorname{BER}_{T}$ (except for high SNR values where the adaptability saturates) and that the ASE is up to $10 \%$ higher in Fig. 4(b), whereas in the case of using $\left\{\hat{\gamma}_{i}^{\nabla}\right\}$, the BER is unnecessarily lower than required (due again to the exponential upper bound employed), and hence, a lower ASE is achieved. We can see that this more precise analysis of the system behavior implies a zero-cost improvement in terms of complexity for the receiver, as the SNR thresholds can be calculated offline in the design stage of the system and their values can be subsequently stored for use during transmission.

3) Application to Systems With Any Antenna Configuration: In this section, we exploit the exact BER expression (23) to analyze systems with different antenna configurations. Fig. 5 shows the ASE for adaptive MIMO transmit beamforming with MRC, mobile speed $v=36 \mathrm{Km} / \mathrm{h}$, and the set of SNR thresholds $\left\{\hat{\gamma}_{i}^{\Delta}\right\}$. As shown in the figure, antenna configurations of, e.g., $3 \times 3$ or $4 \times 4$ can be analyzed with our exact closed-form expression in (23).
ASE $\bar{\nu}[$ bits/symbol]

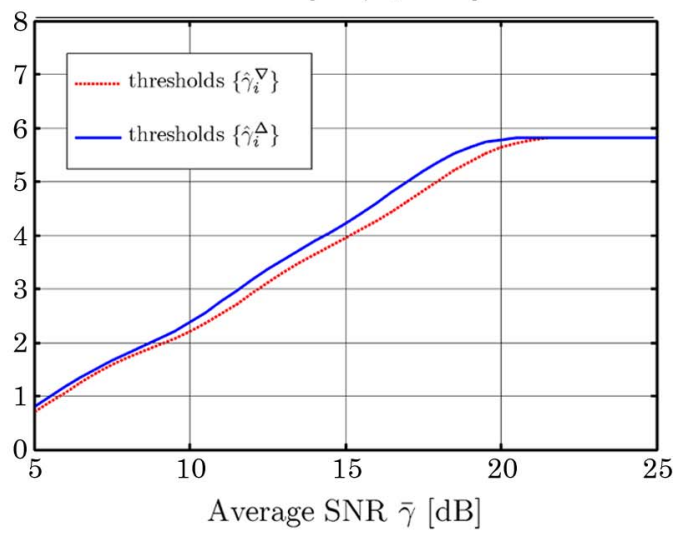

(a)

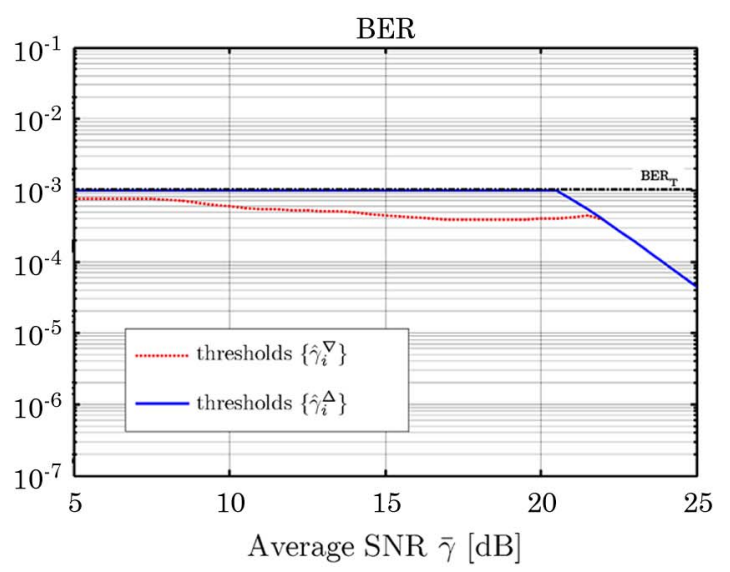

(b)

Fig. 4. (a) ASE and (b) BER for adaptive $2 \times 2 \mathrm{MIMO}$ transmit beamforming with MRC, $v=36 \mathrm{Km} / \mathrm{h}, N_{F}=5$, and $\mathrm{BER}_{T}=10^{-3}$ using thresholds obtained with exact and approximate BER expressions.

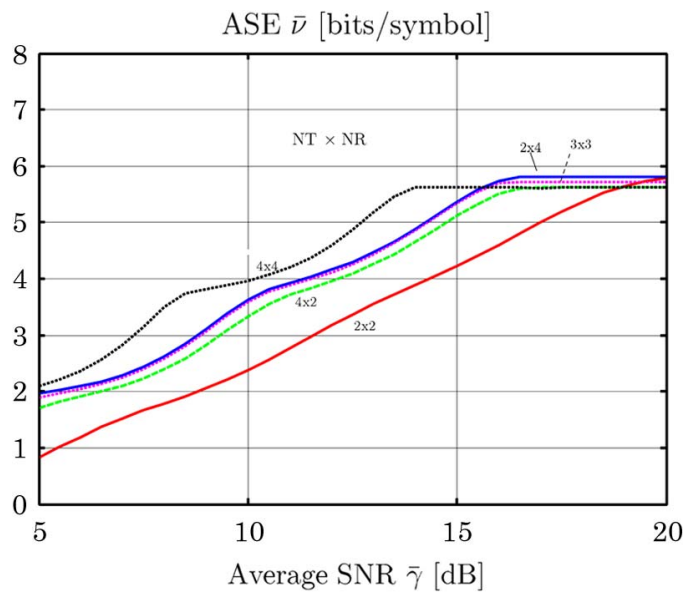

Fig. 5. ASE for different antenna configurations. Adaptive MIMO transmit beamforming with MRC, $v=36 \mathrm{Km} / \mathrm{h}, N_{F}=5$, and $\mathrm{BER}_{T}=10^{-3}$. SNR thresholds obtained with an exact BER expression.

\section{CONCLUSiON}

In this paper, we have addressed a wide family of integrals involving Marcum Q functions and modified Bessel functions that are of interest for performance analysis of adaptive MIMO systems. Then, we have applied our analytical results to obtain 
an exact closed-form BER expression that improves previous analysis of adaptive MIMO transmit beamforming with MRC and prediction errors.

Regarding the analytical results, we have first shown that the incomplete integrals of the type addressed in [11] and [12] are represented by a finite number of elementary functions, generalized Marcum Q functions, and ILHIs. Second, we have proven that the ILHIs are reduced to an expression involving a finite number of modified Bessel and Marcum Q functions. Finally, we have applied these results to obtain an exact closedform expression for the BER of MIMO systems under imperfect CSI that employ adaptive modulation, transmit beamforming, and MRC. As a result, we have obtained an exact closed-form BER expression valid for arbitrary antenna configurations that can be also employed to improve the adaptation policy design at no extra complexity cost.

\section{APPENDIX I \\ PROOF OF LEMMA 1}

As in [11], we perform integration by parts in (3) with

$$
\left\{\begin{array}{l}
u=Q_{n}(a \sqrt{t}, b \sqrt{t}) \\
d u=\frac{b}{2}\left(\frac{b}{a}\right)^{n-1} e^{-\frac{a^{2}+b^{2}}{2} t}\left(a I_{n}(a b t)-b I_{n-1}(a b t)\right) d t \\
v=\left\{[10, \text { eq.2.231-2] }\}=-e^{-\beta t}\left(\frac{m !}{\beta^{m+1}} \sum_{r=0}^{m} \frac{(\beta t)^{r}}{r !}\right)\right. \\
d v=t^{m} e^{-\beta t} d t .
\end{array}\right.
$$

Then, after some algebra, we obtain (4).

\section{APPENDIX II}

\section{PROOF OF LEMMA 3}

Note that $I_{e_{0,0}}(x ; \alpha)=(1 / \alpha) I_{e}((1 / \alpha), \alpha x)$, where $I_{e}$ is the Rice $I_{e}$-function. Then, using the connection between the Rice $I_{e}$-function and the Marcum Q function [3] and after some algebra, we obtain (i). To show (ii), we first apply Lemma 2 with $\nu=0$ and $f(t)=\exp (-\alpha t)$, followed by the substitution of $I_{e_{0,0}}$ by (i). Expression (iii) is obtained using integration by parts with $u=\exp (-\alpha t), d u=-\alpha \exp (-\alpha t) d t, v=$ $I_{0}(t)$, and $d v=I_{1}(t) d t$ and applying (i). Finally, integrating by parts $I_{e_{1,1}}$ with $u=t \exp (-\alpha t), d u=(-\alpha t \exp (-\alpha t)+$ $\exp (-\alpha t)) d t, v=I_{0}(t)$, and $d v=I_{1}(t) d t$ yields

$$
I_{e_{1,1}}(x ; a)=x e^{-\alpha x} I_{0}(x)-I_{e_{0,0}}(x ; a)+a I_{e_{1,0}}(x ; a) .
$$

Substituting (i) and (ii) in (27), (iv) holds, and the proof is complete.

\section{APPENDIX III \\ PROOF OF PROPOSITION 1}

Let us consider the following recursive Luke's formulas for the ILHI of Bessel type [8, pp. 120]:

$$
\begin{aligned}
& \left(\alpha^{2}-1\right) I_{e_{m, n}}(x ; \alpha) \\
& =\alpha(2 m-1) I_{e_{m-1, n}}(x ; \alpha) \\
& \quad+(m-n-1) e^{-\alpha x} x^{m-1} I_{n}(x)-\alpha e^{-\alpha x} x^{m} I_{n}(x) \\
& \quad+\left(n^{2}-(m-1)^{2}\right) I_{e_{m-2, n}}(x ; \alpha)-e^{-\alpha x} x^{m} I_{n+1}(x)
\end{aligned}
$$

$$
\begin{aligned}
& -(n-1) I_{e_{1, n+1}}(x ; \alpha)=-2 n e^{-\alpha x} x I_{n}(x) \\
& -2 n \alpha I_{e_{1, n}}(x ; \alpha)+(n+1) I_{e_{1, n-1}}(x ; \alpha) \\
& -n I_{e_{0, n+1}}(x ; \alpha)=-2 n e^{-\alpha x} I_{n}(x) \\
& -2 n \alpha I_{e_{0, n}}(x ; \alpha)+(n+1) I_{e_{0, n-1}}(x ; \alpha) .
\end{aligned}
$$

First, we show that $I_{e_{0, n}}(x ; \alpha)$ is represented by (6). Such statement for $n=0$ and $n=1$ is obviously true by direct inspection of Lemma 3, where the corresponding coefficients are explicitly given. Let us fix $m=0$ and perform strong induction over $n$ for $n \geqslant 2$. Applying Lemma 3 to (30) and identifying with (6), the following coefficients are obtained for $I_{e_{0,2}(x ; \alpha)}$ :

$$
\left\{\begin{array}{l}
\mathcal{A}_{0,2}^{l}(\alpha)=2 \alpha \mathcal{A}_{0,1}^{l}(\alpha)-\mathcal{A}_{0,0}^{l}(\alpha), \quad l=0,1 \\
\mathcal{B}_{0,2}^{0,0}(\alpha)=2 \alpha \mathcal{B}_{0,1}^{0,0}(\alpha)-\mathcal{B}_{0,0}^{0,0}(\alpha) \\
\mathcal{B}_{0,2}^{0,1}(\alpha)=2
\end{array}\right.
$$

where unspecified coefficients are assumed to be zero. For $n \geqslant$ 2 , applying the induction hypothesis to (30) and identifying with (6) leads to the following coefficients for $I_{e_{0, n}}(x ; \alpha)$ :

$$
\left\{\begin{array}{l}
\mathcal{A}_{0, n}^{l}(\alpha)=2 \alpha \mathcal{A}_{0, n-1}^{l}(\alpha)-\mathcal{A}_{0, n-2}^{l}(\alpha), \quad l=0,1 \\
\mathcal{B}_{0, n}^{0, j}(\alpha)=2 \alpha \mathcal{B}_{0, n-1}^{0, j}(\alpha)-\mathcal{B}_{0, n-2}^{0, j}(\alpha), \quad 0 \leqslant j \leqslant n-2 \\
\mathcal{B}_{0, n}^{0, n-1}(\alpha)=2 \alpha \mathcal{B}_{0, n-1}^{0, n-1}(\alpha)+2 .
\end{array}\right.
$$

Therefore, $I_{e_{0, n}}(x ; \alpha)$ is represented by (6) for $n \geqslant 0$, and the corresponding coefficients are recursively obtained using Proposition 3, (31), and (32). Second, we prove that $I_{e_{1, n}}(x ; \alpha)$ is represented by (6). Again, such statement for $n=0$ and $n=$ 1 is obviously true by direct inspection of Lemma 3. Applying [10, eq. (8.486-1)] to the definition of $I_{e_{1,2}}(x ; \alpha)$, we obtain

$$
I_{e_{1,2}}(x ; \alpha)=I_{e_{1,0}}(x ; \alpha)-2 I_{e_{0,1}}(x ; \alpha) .
$$

Then, using again Lemma 3, the nonzero coefficients for $m=1$ and $n=2$ are

$$
\left\{\begin{array}{l}
\mathcal{A}_{1,2}^{l}(\alpha)=\mathcal{A}_{1,0}^{l}(\alpha)-2 \mathcal{A}_{0,1}^{l}(\alpha), \quad l=0,1 \\
\mathcal{B}_{1,2}^{0,0}(\alpha)=\mathcal{B}_{1,0}^{0,0}-2 \mathcal{B}_{0,1}^{0,0}(\alpha) \\
\mathcal{B}_{1,2}^{1,0}(\alpha)=\mathcal{B}_{1,0}^{1,0}(\alpha) \\
\mathcal{B}_{1,2}^{1,1}(\alpha)=\mathcal{B}_{1,0}^{1,1}(\alpha)
\end{array}\right.
$$

Let us fix $m=1$ and perform strong induction over $n$ for $n \geqslant 3$. Applying the induction hypothesis to (29) and identifying with (6) leads to the following nonzero coefficients for $I_{e_{1, n}(x ; \alpha)}$ :

$$
\left\{\begin{aligned}
\mathcal{A}_{1, n}^{l}(\alpha)= & 2 \alpha \frac{n-1}{n-2} \mathcal{A}_{1, n-1}^{l}(\alpha) \\
& -\frac{n}{n-2} \mathcal{A}_{1, n-2}^{l}(\alpha), \quad l=0,1 \\
\mathcal{B}_{1, n}^{i, j}(\alpha)= & 2 \alpha \frac{n-1}{n-2} \mathcal{B}_{1, n-1}^{i, j}(\alpha) \\
& -\frac{n}{n-2} \mathcal{B}_{1, n-2}^{i, j}(\alpha), \quad \text { for }\left\{\begin{array}{l}
i=0,1 \\
0 \leqslant j \leqslant n-2
\end{array}\right. \\
\mathcal{B}_{1, n}^{0, n-1}(\alpha)= & 2 \alpha \frac{n-1}{n-2} \mathcal{B}_{1, n-1}^{0, n-1}(\alpha) \\
\mathcal{B}_{1, n}^{1, n-1}(\alpha)= & 2 \alpha \frac{n-1}{n-2} \mathcal{B}_{1, n-1}^{1, n-1}(\alpha)+2 \frac{n-1}{n-2} .
\end{aligned}\right.
$$




$$
\left\{\begin{array}{l}
\mathcal{A}_{2, n}^{l}(\alpha)=\frac{3 \alpha}{\alpha^{2}-1} \mathcal{A}_{1, n}^{l}(\alpha)+\frac{n^{2}-1}{\alpha^{2}-1} \mathcal{A}_{0, n}^{l}(\alpha), \quad l=0,1 \\
\mathcal{B}_{2, n}^{0, j}(\alpha)=\frac{3 \alpha}{\alpha^{2}-1} \mathcal{B}_{1, n}^{0, j}(\alpha)+\frac{n^{2}-1}{\alpha^{2}-1} \mathcal{B}_{0, n}^{0, j}(\alpha), \quad 0 \leqslant j \leqslant n+1 \\
\mathcal{B}_{2, n}^{1, j}(\alpha)=\frac{3 \alpha}{\alpha^{2}-1} \mathcal{B}_{1, n}^{1, j}(\alpha)+\frac{n+1}{\alpha^{2}-1} \delta[j-n], \quad 0 \leqslant j \leqslant n+1 \\
\mathcal{B}_{2, n}^{2, j}(\alpha)=\left\{\begin{array}{l}
n=0 \Rightarrow-\frac{\alpha}{\alpha^{2}-1} \delta[j]-\frac{1}{\alpha^{2}-1} \delta[j-1], \quad 0 \leqslant j \leqslant n+1 \\
n>0 \Rightarrow-\frac{\alpha}{\alpha^{2}-1} \delta[j-n]-\frac{1}{\alpha^{2}-1} \delta[j-(n-1)], \quad 0 \leqslant j \leqslant n
\end{array}\right.
\end{array}\right.
$$

$$
\left\{\begin{array}{l}
\mathcal{A}_{m, n}^{l}(\alpha)=\frac{\alpha(2 m-1)}{\alpha^{2}-1} \mathcal{A}_{m-1, n}^{l}(\alpha)+\frac{n^{2}-(m-1)^{2}}{\alpha^{2}-1} \mathcal{A}_{m-2, n}^{l}(\alpha), \quad l=0,1 \\
\mathcal{B}_{m, n}^{i, j}(\alpha)=\frac{\alpha(2 m-1)}{\alpha^{2}-1} \mathcal{B}_{m-1, n}^{i, j}(\alpha)+\frac{n^{2}-(m-1)^{2}}{\alpha^{2}-1} \mathcal{B}_{m-2, n}^{i, j}(\alpha), \quad \text { for } \quad \begin{array}{l}
0 \leqslant i \leqslant m-2 \\
0 \leqslant j \leqslant n+1
\end{array} \\
\mathcal{B}_{m, n}^{m-1, j}(\alpha)=\frac{\alpha(2 m-1)}{\alpha^{2}-1} \mathcal{B}_{m-1, n}^{m-1, j}(\alpha)+\frac{m+n-1}{\alpha^{2}-1} \delta[j-n], \quad 0 \leqslant j \leqslant n+1 \\
\mathcal{B}_{m, n}^{m, j}(\alpha)=\left\{\begin{array}{l}
n=0 \Rightarrow-\frac{\alpha}{\alpha^{2}-1} \delta[j]-\frac{1}{\alpha^{2}-1} \delta[j-1], \quad 0 \leqslant j \leqslant n+1 \\
n>0 \Rightarrow-\frac{\alpha}{\alpha^{2}-1} \delta[j-n]-\frac{1}{\alpha^{2}-1} \delta[j-(n-1)], \quad 0 \leqslant j \leqslant n
\end{array}\right.
\end{array}\right.
$$

Therefore, $I_{e_{1, n}}(x ; \alpha)$ is represented by (6) for $n \geqslant 0$, and the corresponding coefficients are recursively obtained using Lemma 3, (33), and (34).

Finally, we are in a position to show that $I_{e_{m, n}}(x ; \alpha)$ is represented by (6), by fixing $n$ and performing strong induction over $m$ for $m \geqslant 2$. We have proven that such statement is true for $I_{e_{0, n}}(x ; \alpha)$ and $I_{e_{1, n}}(x ; \alpha)$. For $m \geqslant 2$, using [10, eq. (8.486-1)], (28) can be rearranged as follows:

$$
\begin{aligned}
I_{e_{m, n}}(x ; \alpha)= & -\frac{1}{\alpha^{2}-1} e^{-\alpha x} x^{m} I_{n-1}(x) \\
& +\frac{m+n-1}{\alpha^{2}-1} e^{-\alpha x} x^{m-1} I_{n}(x) \\
& -\frac{\alpha}{\alpha^{2}-1} e^{-\alpha x} x^{m} I_{n}(x) \\
& +\frac{\alpha(2 m-1)}{\alpha^{2}-1} I_{e_{m-1, n}}(x ; \alpha) \\
& +\frac{n^{2}-(m-1)^{2}}{\alpha^{2}-1} I_{e_{m-2, n}}(x ; \alpha) .
\end{aligned}
$$

For $I_{e_{2, n}}(x ; \alpha)$, (35) allows obtaining the representation (6) with the coefficients given in (36), shown at the top of the page, where $\delta[\cdot]$ is the Kronecker's delta. For $m \geqslant 2$, the application of the induction hypothesis to (35) and identification with (6) leads to the coefficients in (37), shown at the top of the page, for $I_{e_{m, n}}(x ; \alpha)$. Therefore, $I_{e_{m, n}}(x ; \alpha)$ is represented by (6) for $m \geqslant 0$ and $n \geqslant 0$, and the corresponding coefficients are obtained using Lemma 3, (31)-(34), (36), and (37).

\section{APPENDIX IV}

\section{PROOF OF THE ASYMPTOTIC EXPRESSION (21)}

When $\chi \bar{\gamma} \rightarrow 0$, then $\theta_{p} \rightarrow 0$, and $\eta\left(\theta_{p}\right) \rightarrow 1$. Since coefficients $D(\cdot)$ only depend on $\eta$, it is straightforward to check that $D_{q}\left(\eta\left(\theta_{p}\right) ; N_{R}\right) \rightarrow-(1 / 2) \delta[q]$, where $\delta[\cdot]$ is Kronecker's delta.
Taking these observations into account, if we use the asymptotic relations

$$
\begin{aligned}
Q_{1}(\alpha, \beta) & \sim \sqrt{\frac{\beta}{\alpha}} Q(\beta-\alpha) \text { when } \beta \rightarrow \infty \\
I_{0}(\alpha x) & \sim \frac{\exp (\alpha x)}{\sqrt{2 \pi \alpha x}} \text { when } \alpha \rightarrow \infty
\end{aligned}
$$

then we observe that $b(x)-a(x)=\sqrt{2}((\eta(x)-1) /$ $(\eta(x)+1)), a(x) b(x)=\left(2 \eta(x) /(1+\eta(x))^{2}\right)$, and $\left(a(x)^{2}+\right.$ $\left.b(x)^{2} / 2\right)=\left(\left(1+\eta(x)^{2}\right) /\left(1+\eta(x)^{2}\right)\right)$, and finally substitute in the CCEP (18), the following expression can be obtained:

$$
\begin{aligned}
\mathcal{I}(p ; \hat{\gamma}) \sim \sqrt{\eta\left(\theta_{p}\right)} Q\left(\sqrt{2} \sqrt{\left(\frac{\eta\left(\theta_{p}\right)-1}{\eta\left(\theta_{p}\right)+1}\right)^{2} \frac{\hat{\gamma}}{\chi \bar{\gamma}}}\right) \\
-\frac{1}{2} \frac{\exp \left(-\left(\frac{\eta\left(\theta_{p}\right)-1}{\eta\left(\theta_{p}\right)+1}\right)^{2} \frac{\hat{\gamma}}{\chi \bar{\gamma}}\right)}{\sqrt{2 \pi \frac{2 \eta\left(\theta_{p}\right)}{\left(1+\eta\left(\theta_{p}\right)\right)^{2}}} \frac{\hat{\gamma}}{\chi \bar{\gamma}}} .
\end{aligned}
$$

Using now the asymptotic relation for the Gaussian Q-function $Q(x) \sim\left(\exp \left(-x^{2} / 2\right) / \sqrt{2 \pi} x\right)$ as $x \rightarrow \infty$ in (39), we get the following after some algebra:

$$
\mathcal{I}(p ; \hat{\gamma}) \sim \frac{1}{\sqrt{\frac{4 \pi}{1+\theta_{p}}}} \frac{\exp \left(-\frac{\theta_{p}}{1+\theta_{p}} \frac{\hat{\gamma}}{\chi \bar{\gamma}}\right)}{\sqrt{\frac{\theta_{p}}{1+\theta_{p}} \frac{\hat{\gamma}}{\chi \bar{\gamma}}}} .
$$

Finally, reversing the asymptotic relation for the Gaussian Q-function in (40) and substituting in (17), the asymptotic expression (21) is obtained.

\section{REFERENCES}

[1] M. M. Agrest and M. Z. Maksimov, Theory of Incomplete Cylindrical Functions and Their Applications. New York: Springer-Verlag, 1971.

[2] S. L. Dvorak, "Applications for incomplete Lipschitz-Hankel integrals in electromagnetics," IEEE Antennas Propag. Mag., vol. 36, no. 6, pp. 2632, Dec. 1994 
[3] R. F. Pawula, "Relations between Rice Ie-function and Marcum Q-function with applications to error rate calculations," Electron. Lett., vol. 31, no. 20, pp. 1043-1047, Sep. 1995.

[4] J. I. Marcum, "Table of Q functions," Rand Corp., Santa Monica, CA, U.S. Air Force Project RAND Research Memo. M-339, Jan. 1, 1950. ASTIA Document AD 1165451

[5] A. Nutall, "Some integrals involving the Q function," Naval Underwater Syst. Center, New London, CT, Tech. Rep. 4297, Apr. 17, 1972.

[6] A. Nutall, "Some integrals involving the $Q_{M}$-function," Naval Underwater Syst. Center, New London, CT, Tech. Rep. 4755, May 15, 1974.

[7] G. N. Watson, A Treatise on the Theory of Bessel Functions, 2nd ed. London, U.K.: Cambridge Univ. Press, 1944.

[8] Y. L. Luke, Integrals of Bessel Functions. New York: McGraw-Hill, 1962.

[9] M. Abramowitz and I. A. Stegun, Handbook of Mathematical Functions, 9th ed. New York: Dover, 1970.

[10] I. S. Gradshteyn and I. M. Ryzhik, Table of Integrals, Series, and Products, 6th ed. San Diego, CA: Academic, 2000.

[11] M. K. Simon and M.-S. Alouini, "Some new results for integrals involving the generalized Marcum Q function and their application to performance evaluation over fading channels," IEEE Trans. Wireless Commun., vol. 2, no. 4, pp. 611-615, Jul. 2003.

[12] S. Gaur and A. Annamalai, "Some integrals involving the Qm(a x; b x) with application to error probability analysis of diversity receivers," IEEE Trans. Veh. Technol., vol. 52, no. 6, pp. 1568-1575, Nov. 2003.

[13] A. R. Miller, "Incomplete Lipschitz-Hankel integrals of Bessel functions," J. Math. Anal. Appl., vol. 140, pp. 476-484, 1989.

[14] S. Zhou and G. B. Giannakis, "How accurate channel prediction needs to be for transmit-beamforming with adaptive modulation over Rayleigh MIMO channels," IEEE Trans. Wireless Commun., vol. 3, no. 4, pp. 12851294, Jul. 2004.

[15] J. F. Paris and A. J. Goldsmith, "Adaptive modulation for MIMO beamforming under average BER constraints and imperfect CSI," in Proc. IEEE ICC, Jun. 2006, pp. 1312-1317.

[16] A. Maaref and S. Aissa, "Generalized performance analysis of adaptive PSAM-based transmit-beamforming for wireless MIMO systems," in Proc. VTC, Melbourne, Australia, May 2006, vol. 5, pp. 2578-2582.

[17] A. Maaref and S. Aissa, "Closed-form expressions for the outage and ergodic Shannon capacity of MIMO MRC systems," IEEE Trans. Commun., vol. 53, no. 7, pp. 1092-1095, Jul. 2005.

[18] A. M. Tulino and S. Verdú, Random Matrix Theory and Wireless Communications. Hanover, MA: Now, 2004.

[19] A. J. Goldsmith, Wireless Communications, 1st ed. New York: Cambridge Univ. Press, 2005.

[20] K. Cho and D. Yoon, "On the general BER expression of one- and twodimensional amplitude modulations," IEEE Trans. Commun., vol. 50, no. 7, pp. 1074-1080, Jul. 2002.

[21] J. G. Proakis, Digital Communications, 4th ed. New York: McGrawHill, 2001.

[22] E. Martos-Naya, J. F. Paris, U. Fernandez-Plazaola, and A. J. Goldsmith, "Exact BER analysis for M-QAM modulation with transmit beamforming under channel prediction errors," IEEE Trans. Commun., vol. 7, no. 10, pp. 3674-3678, Oct. 2008.

[23] C. Yuen and B. M. Hochwald, "Achieving near-capacity at low SNR on a multiple-antenna multiple-user channel," IEEE Trans. Commun., vol. 57, no. 1, pp. 69-74, Jan. 2009. [Online]. Available: http://www1.i2r.a-star. edu.sg/ cyuen/publications.html

[24] A. J. Goldsmith and S. G. Chua, "Variable-rate variable-power MQAM for fading channels," IEEE Trans. Commun., vol. 45, no. 10, pp. 12181230, Oct. 1997

[25] S. T. Chung and A. J. Goldsmith, "Degrees of freedom in adaptive modulation: A unified view," IEEE Trans. Commun., vol. 49, no. 9, pp. 15611571, Sep. 2001

[26] C. Kose and D. Goeckel, "On power adaptation in adaptive signaling systems," IEEE Trans. Commun., vol. 48, no. 11, pp. 1769-1773, Nov. 2000.

[27] J. F. Paris, M. C. Aguayo-Torres, and J. T. Entrambasaguas, "Optimum discrete-power adaptive QAM scheme for Rayleigh fading channels," IEEE Commun. Lett., vol. 5, no. 7, pp. 281-283, Jul. 2001.

[28] B. Choi and L. Hanzo, "Optimum mode-switching-assisted constantpower single- and multicarrier adaptive modulation," IEEE Trans. Veh. Technol., vol. 52, no. 3, pp. 536-560, May 2003.

[29] S. Falahati, A. Svensson, M. Sternad, and T. Ekman, "Adaptive modulation systems for predicted wireless channels," IEEE Trans. Commun., vol. 52, no. 2, pp. 307-316, Feb. 2004.

[30] J. F. Paris, M. C. Aguayo-Torres, and J. T. Entrambasaguas, "Nonideal adaptive modulation: Bounded feedback information and imperfect channel estimation," in Proc. IEEE Global Commun. Conf., Dallas, TX, Nov. 2004, pp. 3828-3834.

[31] Z. Zhou and B. Vucetic, "Design of adaptive modulation using imperfect CSI in MIMO systems," Electron. Lett., vol. 40, no. 17, pp. 1073-1075, Aug. 2004.

[32] X. Cai and G. B. Giannakis, "Adaptive PSAM accounting for channel estimation and prediction errors," IEEE Trans. Wireless Commun., vol. 4, no. 1, pp. 246-256, Jan. 2005.

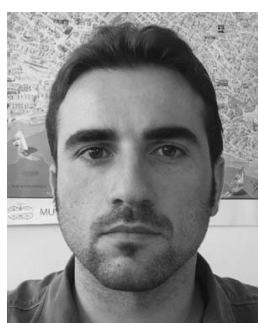

diversity systems.
José F. Paris received the M.Sc. and Ph.D. degrees in telecommunication engineering from Málaga University, Málaga, Spain, in 1996 and 2004, respectively.

From 1994 to 1996, he was with Alcatel, working mainly on the development of wireless telephones. In 1997, he joined Málaga University, where he is currently an Associate Professor with the Communication Engineering Department. His research interests are related to wireless communications, particularly wireless access networks, adaptive modulation, and

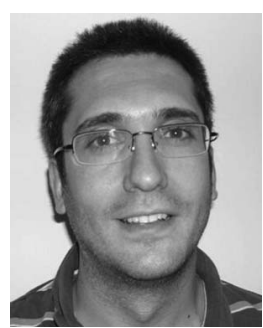

Eduardo Martos-Naya received the M.Sc. and $\mathrm{Ph} . \mathrm{D}$. degrees in telecommunication engineering from Málaga University, Málaga, Spain, in 1996 and 2005, respectively.

In 1997, he joined the Communication Engineering Department, Málaga University, where he is currently an Associate Professor. His research activity includes digital signal processing for communications, primarily synchronization and channel estimation for Power Line Communications and xDSL systems, as well as performance analysis of wireless multiple-input-multiple-output systems.

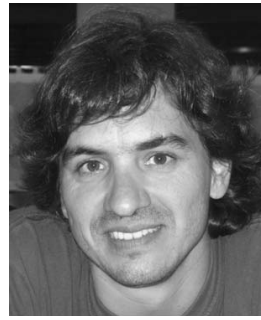

Unai Fernández-Plazaola received the M.Sc. and $\mathrm{Ph} . \mathrm{D}$. degrees in telecommunication engineering from Málaga University, Málaga, Spain, in 1996 and 2005, respectively.

In 1997, he joined Málaga University, where he is currently an Associate Professor with the Communication Engineering Department. His research interests are related to wireless communications and ambiguity-resolution techniques for positioning using global satellite systems.

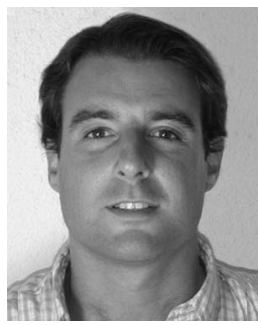

Jesús López-Fernández received the M.Sc. and $\mathrm{Ph} . \mathrm{D}$. degrees in telecommunication engineering from Málaga University, Málaga, Spain, in 1994 and 2001, respectively.

Since 1995, he has been with the Communication Engineering Department, Málaga University, where he is currently an Associate Professor. His research interests are focused on digital signal processing for communications, specifically for xDSL systems. 